\title{
Assessment of Cognitive Performance in Elderly Life via Meaningful Play
}

\author{
Karsten Gielis \\ e-Media Research Lab - KU Leuven, \\ Department of Computer Science \\ Leuven, Belgium \\ karsten.gielis@,kuleuven.be
}

\begin{abstract}
This extended abstract discusses the ongoing research on the "Dr. Solitaire" project. Together with the geriatrics department of the university hospital of Leuven, the possibilities of detecting dementia through tablet games are being explored. A tablet implementation of the popular Klondike Solitaire game was made, capturing digital biomarkers of cognitive performance. At this moment, a study is being designed to discern older adults with Mild Cognitive Impairment from their healthy counterparts.
\end{abstract}

Keywords- Digital Biomarkers, Cognitive Health, Game Analysis, Card Games

\section{INTRODUCTION}

The trend in dementia research has recently shifted to detecting dementia in an earlier stage. This state between normal aging and dementia, called Mild Cognitive Impairment (MCI), is estimated to be prevalent amongst $15 \%$ and $20 \%$ of those above the age of 60 [1]. Detecting dementia in this stage is crucial for disease mitigation and progression[2]-[4]. Moreover, it ensures support for the patient and relatives and helps them in taking decisions for the future[5]-[8].

In this research project, we explore a novel method of detecting and monitoring cognitive impairments, capturing digital biomarker through casual gameplay and by applying machine learning. We hypothesize that by using 'meaningful play', games older adults already enjoy and play, adherence will be better compared to other serious games. Cognitive information is captured through digital biomarkers [9], i.e. game metrics indicative of cognitive performance, for the game Klondike Solitaire. This may provide a new neuropsychological tool, an unobtrusive way of longitudinal monitoring of the patient's psyche, giving them better insight in their cognitive profile. However, using meaningful play to detect cognitive decline has received scant attention in the past. Fortunately, in recent years, more and more attention is given to the subject [10]-[13]. Whilst these studies are well executed and reported, they are sometimes limited in their analysis, relying solely on classic statistical methods, or lacking in complexity of digital biomarkers. Hence, this leaves opportunities for research with fine-grained digital biomarkers and machine learning.

\section{RESEARCH QUESTION}

The main objective of the Dr. Solitaire project is to test the hypothesis that Klondike Solitaire (played on a tablet) can be used to detect MCI. To this end, we focus on answering the following questions: Which cognitive functions are primarily addressed while playing Klondike Solitaire? Which digital biomarkers, found in Klondike Solitaire, are most indicative of cognitive performance? Is it possible to detect cognitive fluctuations [14], the phenomenon of temporary alterations in cognition, attention, and arousal?

\section{RESOURCES AND METHODS}

In earlier work [15], an expert review was conducted to detect the cognitive functions primarily used while playing Klondike Solitaire. Results indicated that attention, executive function, object recognition, abstraction, and memory are addressed while playing Klondike Solitaire. Furthermore, twenty-two players actions were defined indicative of cognitive performance. Strengthened by these results, an Android application was made to capture the aforementioned player actions in the form of digital biomarkers.

Through our contacts with the memory clinic in Leuven and Hasselt, patients with MCI will be recruited. The healthy population will be mobilized through Seniorama, a senior organization from Leuven. We aim to attain 24 participants of each sample. Each participant will undergo one session that comprises of completing a cognitive test battery focused on the cognitive functions stated above and playing three games of Klondike Solitaire. Results of the cognitive tests will be compared to game performance. Furthermore, a statistical analysis will be done to correlate cognitive functions to specific gameplay behavior, providing a deeper understanding of the impact of dementia on casual play. Finally, a thorough analysis of different machine learning models that classify older adults as healthy or cognitively impaired will be performed.

\section{EXPECTED RESULTS}

After this study, we expect to find group differences between older adults suffering from MCI and healthy individuals. Digital biomarkers most indicative of cognitive performance will be defined and linked to their respective cognitive functions. We predict that machine learning algorithms will pick up these differences and are successful in detecting MCI, hence being able to capture the intricacies of cognitive decline. In future research, a study is planned where older adults play the game over a longer period of time. This study will aim to answer the third research question whether cognitive fluctuations can be detected. 


\section{REFERENCES}

[1] R. C. Petersen, "Mild Cognitive Impairment:", CONTINUUM: Lifelong Learning in Neurology, vol. 22, no. 2, Dementia, pp. 404-418, Apr. 2016.

[2] Alzheimer's Association, "2016 Alzheimer's Disease Facts and Figures," 2016.

[3] G. Mushtaq, N. H. Greig, J. A. Khan, and M. A. Kamal, "Status of acetylcholinesterase and butyrylcholinesterase in Alzheimer's disease and type 2 diabetes mellitus," CNS Neurol Disord Drug Targets, vol. 13, no. 8, pp. 1432-1439, 2014.

[4] K. A. Wesnes, D. Aarsland, C. Ballard, and E. Londos, "Memantine improves attention and episodic memory in Parkinson's disease dementia and dementia with Lewy bodies: Memantine in Parkinson's dementia and dementia with Lewy bodies," International Journal of Geriatric Psychiatry, vol. 30, no. 1, pp. 46-54, Jan. 2015.

[5] C. Laske et al., "Innovative diagnostic tools for early detection of Alzheimer's disease," Alzheimer's \& Dementia, vol. 11, no. 5, pp. 561578, May 2015.

[6] B. P. Leifer, "Early Diagnosis of Alzheimer's Disease: Clinical and Economic Benefits," Journal of the American Geriatrics Society, vol. 51, no. 5s2, pp. S281-S288, May 2003.

[7] P. J. Nestor, P. Scheltens, and J. R. Hodges, "Advances in the early detection of Alzheimer's disease,” Nat. Med., vol. 10 Suppl, pp. S34-41, Jul. 2004.

[8] R. C. Petersen et al., "Current concepts in mild cognitive impairment," Arch. Neurol., vol. 58, no. 12, pp. 1985-1992, Dec. 2001.

[9] R. L. Mandryk and M. V. Birk, "The Potential of Game-Based Digital Biomarkers for Modeling Mental Health," JMIR Ment Health, vol. 6, no. 4, p. e13485, Apr. 2019.

[10] P. L. Baniqued et al., "Selling points: What cognitive abilities are tapped by casual video games?," Acta Psychologica, vol. 142, no. 1, pp. 74-86, Jan. 2013.

[11] O. Thompson, S. Barrett, C. Patterson, and D. Craig, "Examining the Neurocognitive Validity of Commercially Available, Smartphone-Based Puzzle Games," Psychology, vol. 03, no. 07, pp. 525-526, 2012.

[12] H. Jimison, M. Pavel, J. McKanna, and J. Pavel, "Unobtrusive monitoring of computer interactions to detect cognitive status in elders," IEEE Trans Inf Technol Biomed, vol. 8, no. 3, pp. 248-252, Sep. 2004.

[13] S. Hagler, H. B. Jimison, and M. Pavel, "Assessing Executive Function Using a Computer Game: Computational Modeling of Cognitive Processes," 2014.

[14] D. R. Lee, J.-P. Taylor, and A. J. Thomas, "Assessment of cognitive fluctuation in dementia: a systematic review of the literature: Cognitive fluctuation in dementia: a systematic review," International Journal of Geriatric Psychiatry, vol. 27, no. 10, pp. 989-998, Oct. 2012.

[15] K. Gielis, F. Brito, J. Tournoy, and V. Vanden Abeele, "Can card games be used to assess mild cognitive impairment? A study of Klondike Solitaire and cognitive functions," in CHI PLAY 2017 Extended Abstracts - Extended Abstracts Publication of the Annual Symposium on Computer-Human Interaction in Play, 2017, pp. 269-276. 